\title{
European Journal \\ of Case Reports in \\ Internal Medicine
}

\section{Leishmaniasis Mimicking Lymphoma and/or Liver Cirrhosis}

\author{
Luca Rossi, Monica Leutner, Daniele Sola, Ettore Bartoli \\ University of Eastern Piedmont "A.Avogadro", Novara, Italy
}

\begin{abstract}
:
A 76-year-old man was admitted to hospital with fever, weight loss, pancytopenia, hepatosplenomegaly and a double monoclonal component IgM-IgG-k, suggesting a diagnosis of myeloma. Bone marrow and liver biopsies disclosed the presence of Donovan bodies, and the titre of anti-Leishmania antibodies was extremely high. After treatment with liposomal amphotericin B, the titre of antibodies fell considerably, while monoclonal components, pancytopenia and clinical symptoms slowly disappeared. Polyclonal $\gamma$-globulins are made of innumerable monoclonal components, one of which can appear as a recognizable band and be misdiagnosed as myeloma when representing the high titre of an antibody directed towards a specific antigen
\end{abstract}

Keywords: Leishmaniasis, multiple myeloma, lymphoma, liver cirrhosis, M-components

Received: $18 / 12 / 2013$

Accepted: $28 / 04 / 2014$

Published: $19 / 05 / 2014$

How to cite this article: Rossi L, Leutner M, Sola D, Bartoli E. Leishmaniasis Mimicking Lymphoma and/or Liver Cirrhosis, EJCRIM 2014;1:doi: 10.12890/2014_000032

Conflicts of Interests: The authors declare that they have no conflicts of interest in this research.

\section{Introduction}

The appearance of monoclonal components in serum focuses attention on multiple myeloma or lymphoma. 


\section{European Journal}

of Case Reports in

Internal Medicine

Pancytopenia associated to spleen/liver enlargement has a broad differential diagnosis, further complicated by its association with a double monoclonal component. We report herein a patient exhibiting these features, in whom the diagnosis could have been missed or delayed if pancytopenia, unreported in the area where the patient was living, had not been taken into consideration, and the $\mathrm{M}$-components had not been linked to the presence of a high titre of antibodies caused by the offending agent.

\section{Case description}

A male Caucasian patient, aged 76, was admitted to an Internal Medicine ward in March 2009 because of worsening dyspnoea, fatigue, and ankle oedema.

The referral diagnosis was monoclonal gammopathy of undetermined significance (MGUS) with double IgG-IgM-k components, increased $p$-globulins and refractory pancytopenia with multi-linear dysplasia (Table 1).

\begin{tabular}{|c|c|}
\hline & $5690 \mathrm{mg} / \mathrm{dl}$ \\
\hline $\mathrm{IgG}$ & $362 \mathrm{mg} / \mathrm{dl}$ \\
\hline $\mathrm{IgA}$ & $210 \mathrm{mg} / \mathrm{dl}$ \\
\hline $\mathrm{P} \mathrm{k}$ & $1400 \mathrm{mg} / \mathrm{dl}$ \\
\hline$\lambda \mathrm{P}$ & $525 \mathrm{mg} / \mathrm{dl}$ \\
\hline $\mathrm{U} \mathrm{k}$ & $42.2 \mathrm{mg} / \mathrm{dl}$ \\
\hline $\mathrm{U} \lambda$ & $27.8 \mathrm{mg} / \mathrm{dl}$ \\
\hline $\mathrm{P} \beta_{2}$ microglobulin & $16,578 \mathrm{ng} / \mathrm{ml}$ \\
\hline $\mathrm{U} \beta_{2}$ microglobulin & $21,565 \mathrm{ng} / \mathrm{ml}$ \\
\hline Daily proteinuria & $417 \mathrm{mg}$ \\
\hline $\mathrm{INR}$ & 1.32 \\
\hline aPTT & $46.8 \mathrm{~s}$ \\
\hline $\mathrm{p} 14$ & + \\
\hline $\mathrm{p} 16$ & + \\
\hline Ab anti-Leishmania & $1 / 5120$ \\
\hline
\end{tabular}

The physical examination disclosed jugular vein swelling, increased central venous pressure (CVP), oedema of the lower limbs, marked painless liver and spleen enlargement, a few spider nevi on his face and no palmar erythema or flapping tremor. Chest, heart and neurologic examinations were normal. His fever spiked daily up to $38^{\circ} \mathrm{C}$. Although repeated blood cultures were negative, he received ceftazidime, amikacin, darbepoietin and granulocyte colony stimulating factor (GCS-F). Results of the critical blood exams are reported in Table 1.

The bone marrow biopsy showed increased cellularity, and marked interstitial and perivascular plasma cell infiltration, positive to both $\kappa$ and $\lambda$ chains, organized in small clusters and amounting to $35 \%$ of all marrow cells. There was evidence of dyserythropoiesis, occasional micromegakaryocytes and an important fibrotic argyrophilic reticulum.

Table 1: Data obtained on patient's

admission, referring to measurements

indicated by the international

nomenclature, as explained in the text.

These findings were interpreted as suggestive of myelodysplasia associated to plasmacytosis and marrow 


\section{European Journal \\ of Case Reports in \\ Internal Medicine}

fibrosis (Fig. 1).

Nuclear magnetic resonance (NMR) disclosed increased liver size, left lobe hypertrophy, portal vein of $20 \mathrm{~mm}$ diameter and spleen of $20 \times 20 \mathrm{~cm}$.

Liver elastometry measured a stiffness of $27.7 \mathrm{KPa}$, compatible with liver cirrhosis.

Liver biopsy evidenced pericellular and perisinusoidal fibrosis, with regenerative nodules, and a polymorphous portal and periportal inflammatory infiltrate, formed by granulocytes and macrophages (Fig. 2).

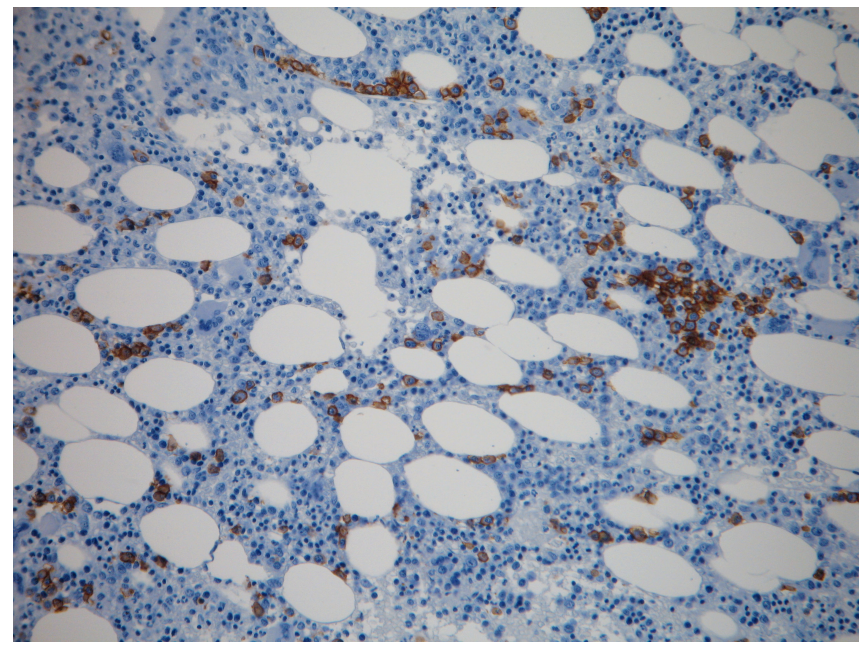

Figure 1: Bone biopsy. The interstitial plasmocytosis is shown by the histochemical reaction with anti-CD138 antibody, revealed by the rusty colour (magnification 20x, DAB/haematoxylin stain). For explanation see text.

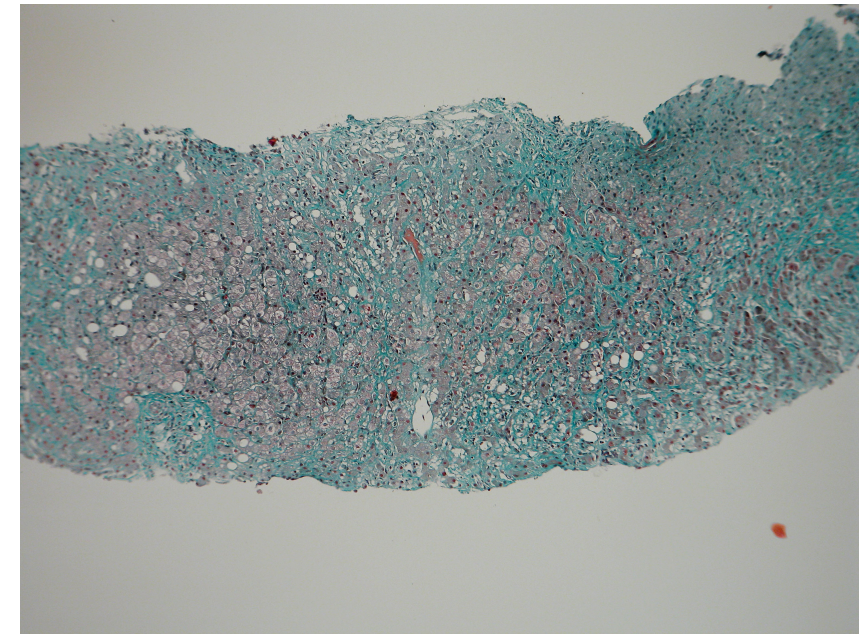

Figure 2: Liver biopsy. Active septae are evident, subdividing nodules with peripheral necrosis and cell ballooning. One nodule has been invaded by fibrotic tissue isolating single cells/groups of cells. The sinusoidal and pericellular fibrosis with active septae is suggestive of chronic active hepatitis evolving towards cirrhosis (magnification 10x, Trichromic Masson stain).

At higher magnification, numerous Donovan bodies were recognized inside liver cells and macrophages (Fig. 3). The bone marrow biopsy, re-examined, also showed Donovan bodies inside histiocytes (Fig. 4). The titre of anti-Leishmania antibodies was executed at the "Istituto Superiore di Sanità", Rome (Italy), and was found positive at a 1/5120 dilution, associated to a strong positivity to $\mathrm{p} 14$ and weak positivity to $\mathrm{p} 16$ by Western blot. Leishmaniasis is unreported in residents in Piedmont (Italy). However, the patient spent the summer months in the county of Savona (Liguria, Italy), where an epidemic source of infection is known and Phlebotomus papatasi, a vector insect of Leishmania, is present along the coast. The patient was treated with liposomal amphotericin B (Ambisome) $5 \mathrm{mg} / \mathrm{kg} /$ day for 5 consecutive days and then once weekly for 5 weeks.

The disease remitted completely: IgG fell to 1430 , IgM to 115 , IgA to $220 \mathrm{mg} / \mathrm{dl}$ and antibody titre to $1 / 2520$. The hepatosplenomegaly reverted to normal, and so did the abnormal liver exams, while pancytopenia 
disappeared.

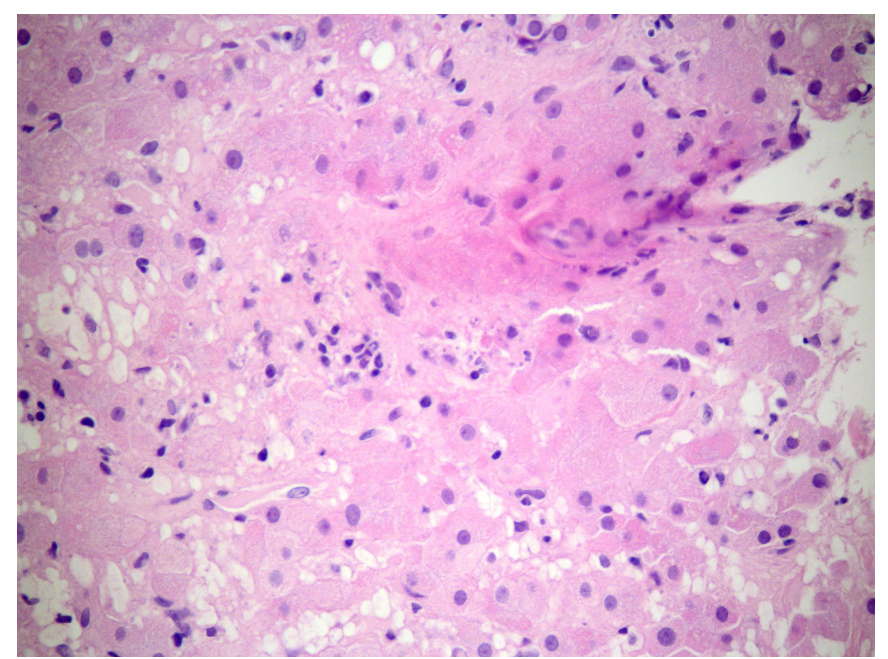

Figure 3: Liver biopsy. Ballooned liver cells and macrophages contain several Donovan bodies in their cytoplasm (100x, Giemsa).

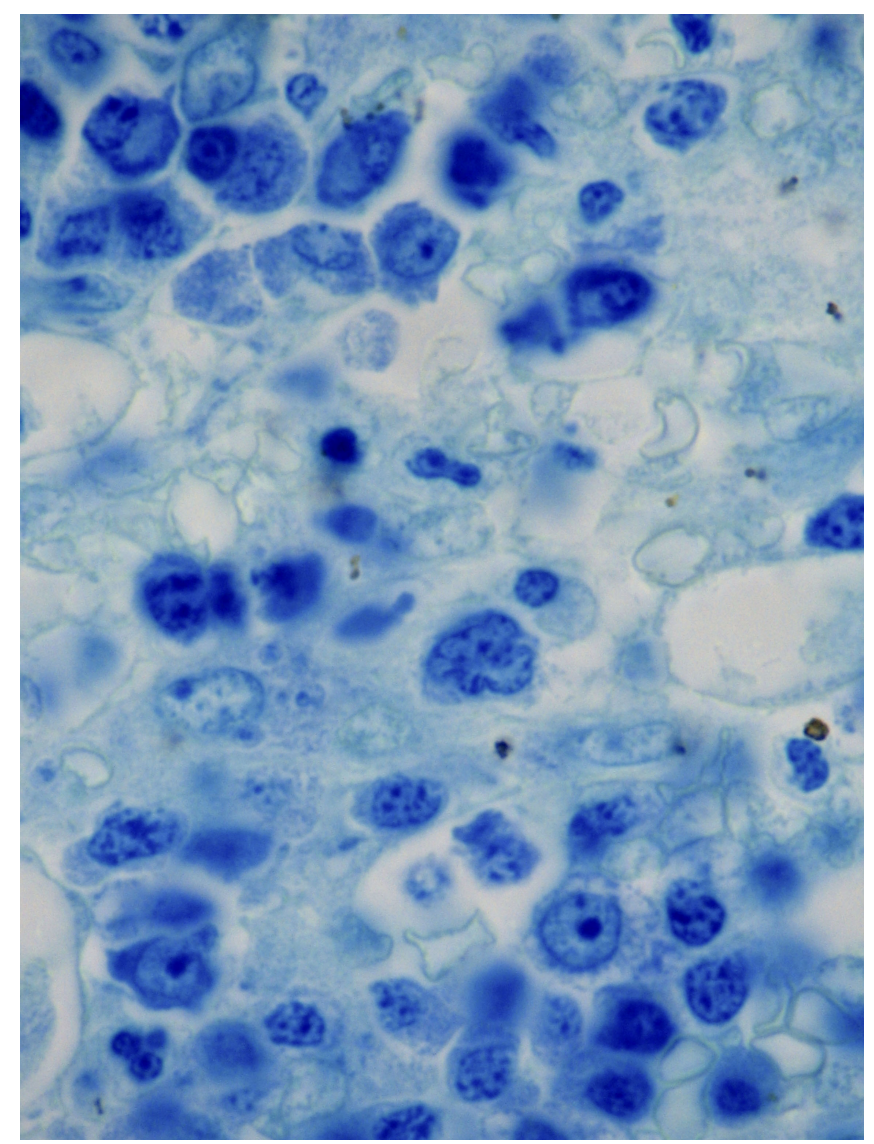

Figure 4: Bone marrow biopsy. Rare macrophages and/or reticulum cells contain several Donovan bodies in their cytoplasm (100x, Giemsa).

\section{Discussion}

The differential diagnosis of this patient was difficult, as several entities are characterized by hepatosplenomegaly, pancytopenia fever and a double IgM-IgG monoclonal component. Felty's syndrome was unlikely because of the absence of rheumatoid arthritis ${ }^{1}$, while a T-hepatosplenic lymphoma, although rare, was possible. However, this and angioimmunoblastic lymphoma are aggressive, leading to a progressive disruption of liver structure, attended by jaundice and ascites. In contrast, the present patient displayed a more chronic course, more typical of T-large granular lymphocytes leukaemia, a disease associated with rheumatoid arthritis and red cell aplasia, which were not present in this case. Hairy cell leukaemia (HCL) fitted the picture, but hairy cells were not found in his peripheral blood. The monoclonal components were suggestive of other B-cell lymphomas, namely prolymphocytic leukaemia, because of liver/spleen involvement. However, the typical plasmacytoid lymphocytes were not observed in the patient's peripheral blood.

Hepatosplenic T-cell lymphoma (HSTL) is a neoplasm of mature T cells that infiltrates the sinusoids of the spleen, 
liver and bone marrow ${ }^{2}$. Most patients exhibit marked hepatosplenomegaly without lymphadenopathy at physical examination, and the blood exams demonstrate thrombocytopenia. Other common findings include systemic B symptoms (weight loss, fever, night sweats), anaemia, neutropenia and abnormal LFTs. The diagnosis is based on the accumulation of malignant atypical lymphocytes expressing CD2/CD3/CD7/CD16, $\gamma / \delta$-T-cell receptors and/or $\alpha / \beta$-T-cell-receptors. This condition was ruled out because of the absence of the typical histological pattern.

Angio-immunoblastic T-cell lymphoma (AITL) is one of the more common peripheral T-cell lymphomas and is thought to come from peripheral CD4-positive T cells, perhaps corresponding to a subset of follicular helper T-cells. Patients typically present with acute onset of generalized lymphadenopathy, hepatosplenomegaly and systemic B symptoms with or without a rash, occasionally associated with autoimmune haemolytic anaemia, plasmacytosis or polyclonal hypergammaglobulinaemia. The diagnosis of AITL is best made by excisional biopsy of a lymph node ${ }^{3}$. These two last entities are aggressive, leading to a progressive disruption of liver structure, attended by jaundice and ascites, whereas the present patient displayed a more chronic course.

T-large granular lymphocyte (LGL) leukaemia is a clonal disease of the large granular lymphocyte characterized by peripheral blood and marrow lymphocytic infiltration with LGLs, splenomegaly and neutropenia. LGL leukaemia can be of T- or NK-cell lineage ${ }^{4}$. Even though monoclonal gammopathy of undetermined significance (MGUS) and multiple myeloma have been described in association with LGL leukaemia, this disease was ruled out because it requires association with rheumatoid arthritis and red cell aplasia.

$\mathrm{HCL}$ causes splenomegaly (which may be massive), systemic complaints, bruising and bleeding secondary to severe thrombocytopenia, and recurrent infections secondary to granulocytopenia and monocytopenia. A diagnosis of $\mathrm{HCL}$ is confirmed when the abnormal cells display pan-B cell antigens (e.g. CD19/CD20/CD22) along with positivity for $\mathrm{CD} 103 / \mathrm{CD} 11 \mathrm{c} / \mathrm{CD} 25^{5}$. The clinical features $\mathrm{HCL}$ fitted our patient, but hairy cells were not found in his peripheral blood.

Prolymphocytic leukaemia (B-PLL) is a rare B-cell neoplasm comprising prolymphocytes, typically with involvement of the peripheral blood, bone marrow and spleen. It is most common in elderly Caucasians. A T-cell variant has been reported ${ }^{2}$.

Falciparum malaria, nowadays unheard of in Italy in people who have not travelled to endemic areas, was ruled out by repeated peripheral blood smears. Mycobacterium avium complex is being reported in HIV-positive patients ${ }^{6}$, but the patient was negative. Typhoid fever was ruled out by the negative serum reaction and the absence of roseola. Brucellosis causes leukocytosis ${ }^{7}$, whereas the patient was leukopenic. Peripheral leukocytosis could have been expected also in portal vein phlebitis or abscess formation in the context of liver cirrhosis $^{8}$. Having ruled out all the above possibilities, biclonal multiple myeloma was the only possibility left, although this constitutes only $1 \%$ of all myelomas ${ }^{9}$. However, the progressive liver disease and worsening of clinical conditions, pancytopenia, spleen enlargement, fever with marked sweating and clinical signs suggestive of a systemic infection called for a unifying infectious hypothesis. Lieshmaniasis, though unheard of in this 


\section{European Journal}

of Case Reports in

Internal Medicine

geographic area, did satisfy the clinical/laboratory picture, with the exception of the monoclonal bands, unless these were due to a markedly high titre of anti-Leishmania antibodies, detected during the switch from IgM to $\lg$.

In conclusion, although leishmaniasis in northern Italy is increasingly reported only in travellers from endemic areas and countries ${ }^{10}$, and is thus not usually suspected, it was indeed the correct diagnosis in this patient. The monoclonal bands, a "red herring" in the differential diagnosis, were in fact due to the high titre of specific anti-Leishmania antibodies, "frozen" in the blood sample during the switch from IgM to IgG.

This case demonstrates how an apparently clear diagnosis of B-cell lymphoma (including initial multiple myeloma or Waldenström's disease) and/or liver cirrhosis could mimic a reversible condition.

After all, "polyclonal" $v$-globulins are made of an almost unlimited number of small monoclonal components, each specific for an offending agent. It should come as no surprise that, during the acute antibody response to an antigen, the monoclonal component could be detected, suggesting multiple myeloma or lymphoma. This M-component should disappear with the drop in antibody titre, as happened in our patient. In fact, a similar case due to Babesia infection has been previously reported, suggesting that protozoan infections may be more likely to cause pseudo-myeloma or -lymphoma.

\section{Learning Points}

- Rare diseases, although rare, do occur, even when not included in the differential diagnosis.

- The differential diagnosis of fever, leucopoenia and liver/spleen enlargement includes leishmaniasis.

- A double IgM-IgG monoclonal antibody should raise suspicion of a recent severe infection.

- There are cases of apparent liver cirrhosis, with highly suggestive elastometry stiffness values, which are reversible.

\section{References}

1. Hofbauer LC, Diebold J, Heufelder AE. Rheumatoid arthritis, neutropenia and splenomegaly: the Felty syndrome, Deutsch Med Wochenschr 1995;120:1689-1694.

2. Swerdlow SH, Campo E, Harris NL, Jaffe ES, Pileri SA, Stein H, Thiele J, et al. World Health Organization Classification of Tumours of Haematopoietic and Lymphoid Tissues, Lyon: IARC Press, 2008.

3. Dogan A, Attygalle AD, Kyriakou C. Angioimmunoblastic T-cell lymphoma, Br J Haematol 2003;121:681.

4. Lamy T, Loughran TP Jr (2003) Clinical features of large granular lymphocyte leukemia, Semin Hematol 2003;40:185.

5. Jaffe ES, Harris NL, Stein H, Vardiman JW. World Health Organization Classification of Tumours. Pathology and Genetics of Tumours of Haematopoietic and Lymphoid Tissues, Lyon: IARC Press, 2001. 


\section{European Journal}

of Case Reports in

Internal Medicine

6. Corti M, Palmero D. Mycobacterium avium complex infection in HIV/AIDS patients, Exp Rev Anti Infect Ther 2008;6:351-363.

7. Glynn MK, Lynn TV. Brucellosis. Veterinary medicine today: zoonosis update, J Am Vet Med Assoc 2008;233:900-908.

8. Chirinos JA, Garcia J, Alcaide ML, Toledo G, Baracco GJ, Lichtstein DM. Septic thrombophlebitis: diagnosis and management, Am J Cardiovasc Drugs 2006;6:9-14.

9. Mahto M, Balakrishnan P, Koner BC, Lali P, Mishra TK, Saxena A. Rare case of biclonal gammopathy. Int J Case Rep Images 2011;2:11-14 .

10. Gaeta GB, Gradoni L, Gramiccia M et al. Leishmaniosi viscerale in Italia. Epidemiologia, clinica, terapia, Rec Progr Med 1994;85:340-346. 